\title{
Li Abundance in Pop I Stars
}

\author{
Luca Pasquini \\ European Southern Observatory, Karl Schwarzschild Strasse 2 \\ 85748 Garching bei München, Germany
}

\begin{abstract}
.
The study of Li in Pop I stars has focussed in the last years on observations of open clusters, spanning a large range of ages and metallicities. So far the observational picture is quite complex: the data indicate several phenomena which are not well understood, from the 'Li dip', to the scatter in $\mathrm{Li}$ abundances found among (otherwise similar) stars both in young and old clusters. Models fail in reproducing most of the observed features; in particular the almost total lack of dependence of Li depletion with metallicity. The comparison between clusters of different ages show that no PMS depletion occurs, and that for a large fraction of old star no additional depletion occurs after the first $\sim 1.5$ Gyrs, while the depletion is strong at earlier ages. These observations are crucial for our understanding of the relationship between the Pop II Li Plateau and the Li primordial abundance.
\end{abstract}

\section{Introduction}

After the pioneering work of Spite and Spite (1982), the current paradigm is that while Pop II (metal poor) stars give (or are close to) the primordial Li abundance, Li evolution in Pop I stars can be used to trace the Li galactic enrichment and the phenomena occurring in the stellar interiors. It is however clear that it is definitely uncomfortable to use the Pop II (Spite) Li plateau as a signature of the primordial $\mathrm{Li}$ abundance as long as we do not understand how $\mathrm{Li}$ is produced in the Galaxy and depleted in stars. The study of Li in Pop I stars is therefore also relevant to the measurement of its primordial abundance.

In this review I will concentrate on observations of solar MS stars i.e. stars cooler than $6200 \mathrm{~K}$ and warmer than $5000 \mathrm{~K}$, therefore not discussing either the whole 'Li dip' problem (Boesgaard and Tripicco 1986) or the interesting lower MS stars (Martin 2000, this volume). Although with a somewhat different approach, excellent recent reviews can be found in Jeffries 2000, Delyiannis 2000. In recent years most observations have been carried out among open cluster stars. The main reason for studying in detail open clusters is very simple: they represent the best approximation of a simple, homogeneous population: by studying them and covering an as wide as possible stellar parameter space (temperature, metallicity, age) we will be able to disentangle the dependence of $\mathrm{Li}$ evolution on stellar parameters. The optimization of high resolution spectrographs coupled to 4 and 8 meter telescopes has contributed to the observations of the often faint 
Table 1. Summary of recent open clusters Li observations. For an explanation of the columns see the text.

\begin{tabular}{l|l|l|l|l|l|}
\hline \multicolumn{1}{c}{ OC } & Age & \multicolumn{1}{c}{ Fe/H } & \multicolumn{1}{c}{ Rstar } & Ref. \\
\hline \hline NGC 2264 & $6.6^{*}$ & -0.15 & 6,28 & FG, GK & K1998, S99* \\
IC2602 & 7.36 & $-0.1^{*}$ & 25,26 & FGKM,GKM & R97a,M99* \\
IC4665 & 7.58 & $?$ & 14 & GKM & MM97 \\
IC2391 & 7.64 & $-0.1^{*}$ & 10,22 & GKM, GKM & S89,M99* \\
Blanco 1 & 7.70 & $0.12^{*}$ & 39,17 & FGK, GK & PO97,JJ99* \\
NGC2516 & 7.79 & 0.06 & 24 & FGK & J98 \\
NGC2547 & 7.88 & -0.16 & 34 & KM & J99 \\
$\alpha$ Per & 7.90 & 0.1 & $5,3,29,18$ & F,M,FGK,KM & B88,GL94,B96,R98 \\
Pleiades & 7.92 & $-0.034^{*}$ & $17,95,13,15,8$ & F,FGK,K,KM,GK & B88,S93,GL94,J96,R96 \\
\hline \hline M35 & 8.0 & -0.17 & 39 & GK & BN99 \\
NGC6475 & 8.11 & +0.08 & 35,49 & F,G,K & JJ97,R99a \\
NGC1039 & 8.26 & -0.26 & 34 & FGK & J97 \\
\hline \hline Coma & 8.69 & -0.03 & $16,5,15,11$ & F,FG,FGK,GK & B87,S90,J99a,F99 \\
NGC6633 & 8.66 & 0.0 & 21 & FGK & Je97 \\
Hyades a & 8.92 & 0.12 & $32,14,23,68,12$ & F,F,FGK,K & B87,BB88,S90,T93,S95 \\
Praesepe a & 8.92 & 0.07 & 63 & FG & S93 \\
\hline NGC752 a & 9.15 & -0.16 & 19,6 & FG,F & HP86,HP88 \\
NGC3680 a & 9.18 & -0.16 & 11 & FGK & P98,R00 \\
IC4651 a & 9.25 & -0.16 & 14 & FGK & R00 \\
NGC2243 a & 9.75 & -0.56 & 11 & FG & H99 \\
M67 a & 9.80 & -0.09 & $7,6,14,25$ & FG,F,FG,FG & HP86a,S87,P97,J99b \\
NGC188 a & 9.86 & -0.05 & 7 & F & HP88a \\
\hline \hline
\end{tabular}

open cluster stars. The results are impressive: from a total of 17 studies on 9 Clusters, covering less than 60 stars in clusters in addition to the well studied Hyades, Praesepe and Pleiades published prior to 1994, we have reached the present situation, summarized in Table 1, where more than 40 studies have been carried out on a total of 22 Clusters, with observations of more than 400 stars in the 19 clusters in addition to the 3 classic ones. In Table 1 a summary is given: in addition to the cluster name, age is given (following Mermillod scale for the young clusters, the Friel scale for the old ones) metallicity (when spectroscopic determination is available, this has been preferred and they are marked with * in Table 1), number of stars observed/work, their spectral type and an abbreviation for the work, while full references are given in the reference section. Although an effort for completeness has been performed, I apologize if references are missing. In the following of the paper the references given in Table 1 are not repeated when used; note that all the figures show data brought to the same temperature and $\mathrm{Li}$ abundance scales.

By using this impressive set of data (which is likely to grow rapidly in the next years, thanks to the use of the new generation of multi object high resolution spectrographs, see e.g. Pasquini 2000) we can start to answer to the following questions:

1. Does Pre Main Sequence (PMS) depletion occur? 
2. Does Main Sequence (MS) depletion occur? How large is this MS depletion? On which timescales does it occur? Does it dependence on metallicity? On other parameters?

3. Is the Li abundance uniquely determined by stellar macroscopic parameters like $\mathrm{T}_{\text {eff }}$, Mass, Age, Composition?

\section{PMS depletion}

To know if strong PMS depletion occurs among solar stars is relevant because most evolutionary models predict a strong Li PMS depletion, and if this would occur, it should be taken into account when interpreting the Plateau as primordial Li. Figure 1 shows Li vs. effective temperature for three young clusters, together with the slightly older Pleiades. IC2602, IC2391, IC4665 span a range of a few million years in age; their G stars have just arrived on the MS, while the cooler K stars are at the end of the PMS phase, approaching the MS. They have different metallicity, although the range spanned is admittedly small. Considering the original solar system value $\mathrm{N}(\mathrm{Li})=3.3$ and that the same value is found today in PMS stars after proper NLTE correction, it is clear from Figure 1 that the $\mathrm{G}$ stars in these clusters, with $\mathrm{N}(\mathrm{Li}) \sim 3.2$, have experienced hardly any PMS depletion, with no dependence on metallicity. The situation is different for K Stars with effective temperatures below $\sim 5000 \mathrm{~K}$ : among them in fact a rather strong depletion is observed: since they have not yet reached the MS this depletion occurred in the PMS phase. It is finally worth noticing that almost no differences in Li abundance exist between these clusters and the older Pleiades despite their 60-90 Myr younger age.

\section{Main Sequence Depletion}

The question of whether MS Li depletion occurs and how, is intimately linked to the physical mechanisms present in the stellar interior and to the mixing acting at the basis of the convective zone. It is worth remembering indeed that standard models predict for the Sun that the bottom of the convective zone is not deep enough to produce any Li burning; in addition, still according to these models, a Li depletion should strongly depend on stellar metallicity: more metal poor stars should suffer a much lower depletion (Pinsonneault et al. 1989, Swensson 1995). It is also worth pointing out that in all non-standard models (i.e. those including interior extra mixing, either produced by diffusion, rotation or gravity waves) the extra depletion predicted is really 'extra', that is, it should apply in addition to the depletion predicted by the standard ones; this implies that some of the basic features of the standard models should still hold (Jeffries 2000). In Figure 2 the data for 4 clusters of different ages are shown: Hyades (600 Myr), IC4651 and NGC3680 (1.6 Gyrs) and the 4 Gyrs old M67.

It is clear that, YES, main sequence Li depletion is indeed occurring. In particular, when comparing the Hyades with the younger Pleiades (cfr. Figure 1) in the $\sim 500$ Myrs between the two clusters a depletion of $\sim 0.4$ dex had occurred for the G stars. This MS depletion, on the other hand, tends to become much smaller with an increase in stellar ages: the comparison of the two intermediate 


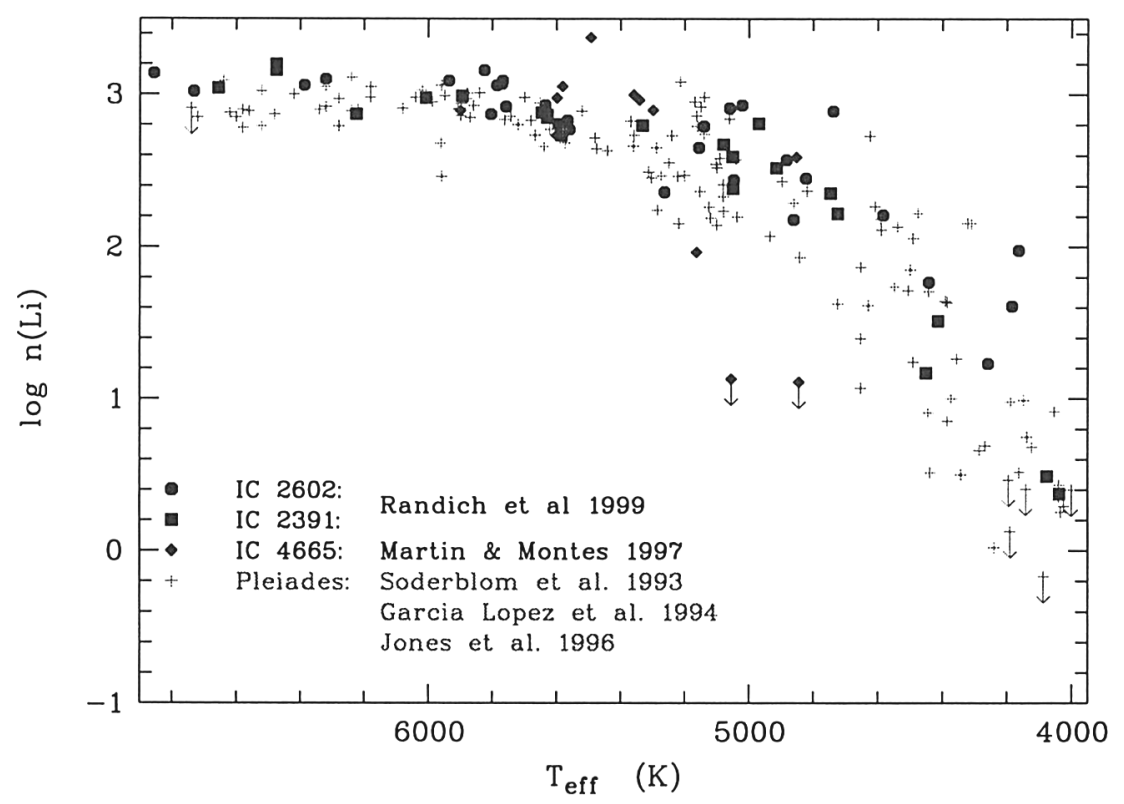

Figure 1. Li vs. effective temperature for three very young open clusters and the Pleiades

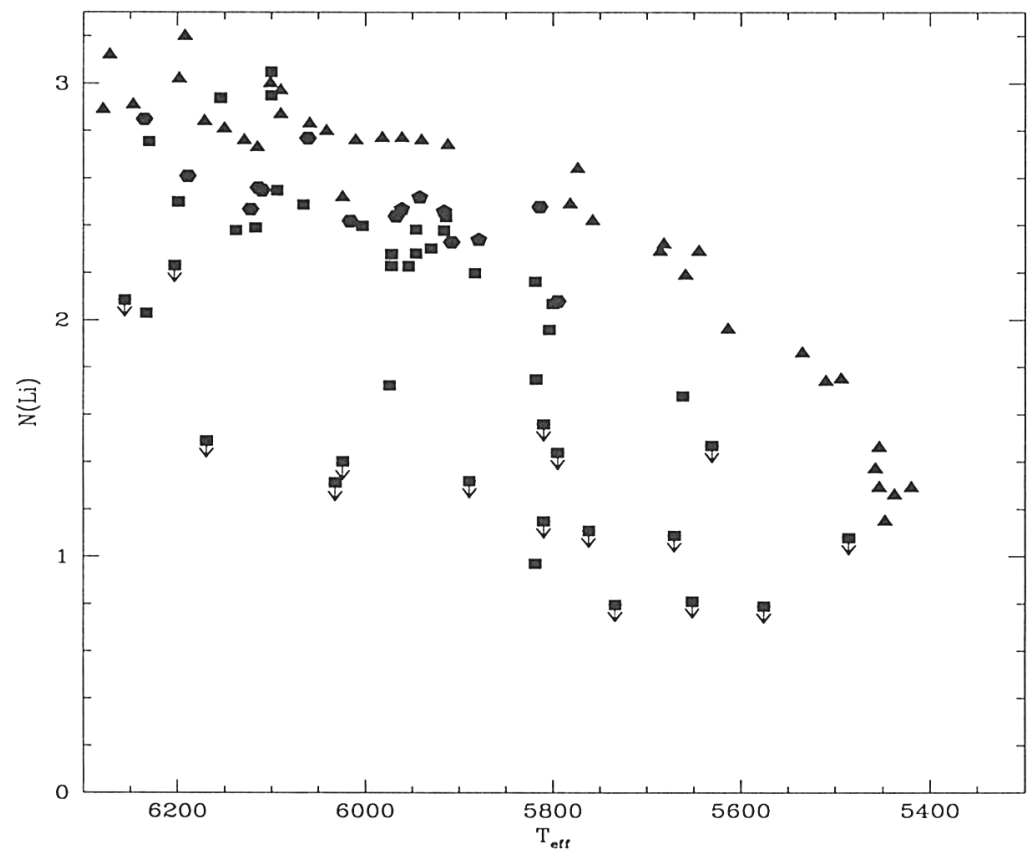

Figure 2. Li vs. effective temperature for Hyades (600 Myr, triangles), IC4651 and NGC3680 (1.6 Gyrs, pentagons and hexagons), M67 (4 gyrs, squares) 


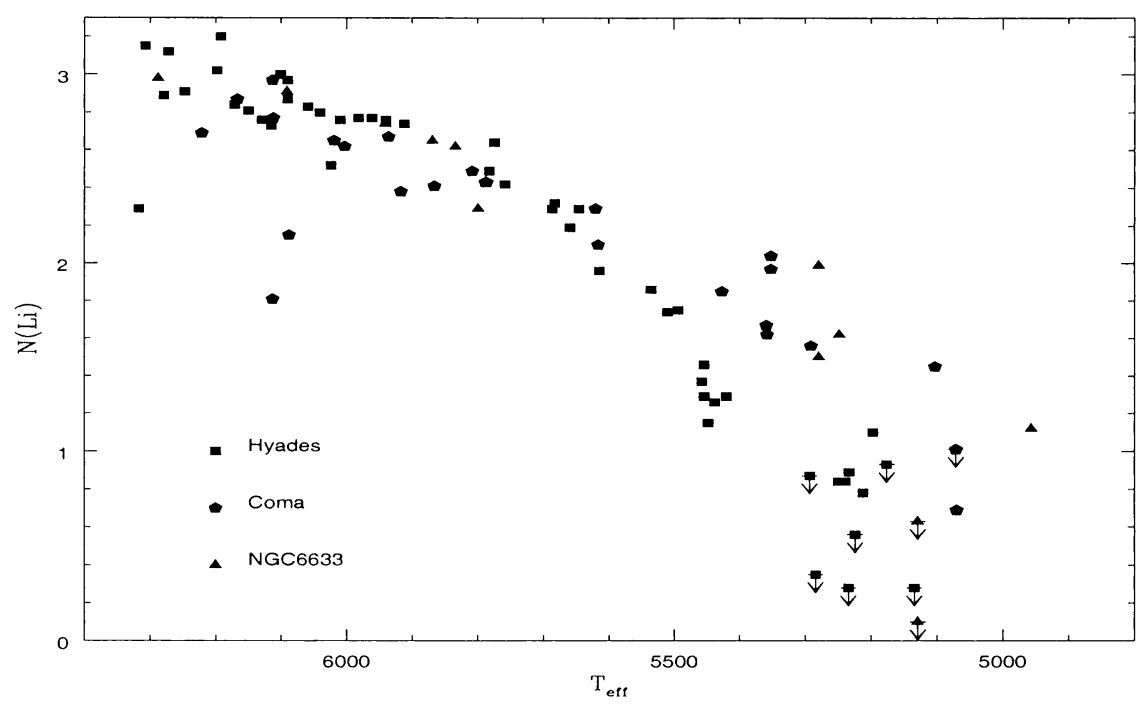

Figure 3. $\mathrm{Li}$ vs. effective temperature for three clusters with same age but different metallicity: Hyades $([\mathrm{Fe} / \mathrm{H}]=0.15)$, NGC6633 $([\mathrm{Fe} / \mathrm{H}]=0, \mathrm{Coma}([\mathrm{Fe} / \mathrm{H}]=-0.03)$; no appreciable difference is observed among G stars; the effect of metallicity may be present among the K stars

age clusters IC4651 and NGC3680 with the Hyades shows that in the subsequent 1 Gyr only a very small amount of Li depletion occurs, of the order of 0.2 dex. The direct comparison between the intermediate age clusters and the upper envelope M67 stars, on the other hand, shows that for a large fraction of stars NO additional depletion between 1.6 and 4 Gyrs occurred.

\subsection{Dependence on Metallicity?}

The most relevant stellar parameter expected to determine depletion is stellar metallicity; to investigate this point in Figure 3 the Li data for 3 clusters with similar age but substantially different metallicity are shown: Coma, NGC6633 and the Hyades. Since, as shown above, most main sequence depletion has occurred at their age, this should be a rather stringent test. From Figure 3 it is evident that for $\mathrm{G}$ stars very little dependence exists on metallicity, if at all.

The situation could be different for the cooler K stars: pending more and more accurate data, for these stars a slight dependence on metallicity seems to be present, although at a much lower level than expected by theory.

One has to caveat, on the other hand, that according to several models stellar metallicity (which is here considered as given by Fe and a solar element pattern is assumed) is not the only relevant abundance, but $\mathrm{Li}$ depletion may be very sensitive to the abundance of elements like $\mathrm{O}, \mathrm{Mg}, \mathrm{Si}$ (Swenson et al. 1994). Unfortunately for most clusters these abundances are unknown and it would be extremely important to gather them soon. 


\subsection{Dependence on Rotation?}

Stellar rotation is one of the parameters typically ignored in evolutionary models of low main sequence stars, but for the specific case of Li evolution, it may have a substantial role, either through meridional circulation (e.g. Vauclair, these proceedings) or rotationally induced mixing (e.g. Pinsonneault, these proceedings). The assessment of the relevance of rotation is not an easy task, because the driving parameter is expected to be the rotational history of the star, rather than its present, measured Vsini value.

The strongest case for the case of stellar rotation is given by short period binaries in open clusters (Delyiannis, these proceedings); in Figure 2, for instance, the M67 stars with the highest $\mathrm{Li}$ abundance refer to a short period binary system. Short period binaries are typically tidally locked, therefore their rotational period is much shorter than the corresponding one of single stars, which spin down during their main sequence lifetime.

Another strong case for rotation was presented by Soderblom et al. 1993, in their study of the Pleiades: among Pleiades K stars they found a large spread in Li abundances; and they showed that statistically the fast rotator $\mathrm{K}$ stars in the Pleiades have higher Li abundances than the slow rotating ones.

Unfortunately I believe that the arguments so far presented are not conclusive, on purely observational grounds.

Definitely more spectra of cluster short period binaries are required to ensure a proper sample; in M67, for instance, many exist and the case presented so far is not completely clear (cfr. the discussion in Pasquini et al. 1997).

Concerning the Pleiades K stars, the case of dependence on rotation is not at all clear: for these stars Soderblom et al. (1993) and Jeffries (1999) acquired potassium spectra. The potassium resonance lines are an important crosscheck, because these lines are formed in the same layers of the $\mathrm{Li}$ resonance doublet. In Figure 4 the equivalent widths of the potassium lines vs. the Li lines are shown for the early $\mathrm{K}$ stars in the Pleiades. If the spread of $\mathrm{Li}$ abundance could be explained with differential depletion, for the potassium lines this argument does not apply, since potassium is not burnt at low temperatures. Some of the scatter can be given by measurement errors (the equivalent widths measured by Jeffries and Soderblom for stars in common may vary by more than $100 \mathrm{~m} \AA$ ), but it may also be that for these rapid rotators our models are inadequate to represent their atmospheres, or for instance, that the effective temperatures derived by colors are not appropriate. I believe that this point should be addressed as early as possible. Similar conclusions were reached by King et al (2000), who also found the same problem in the analysis of the potassium and Li lines, but in addition, found a possible dependence of the potassium line strength with a chromospheric index derived from the Ca II triplet.

Finally, the $4 \mathrm{G}$ stars belonging to NGC3680 have rather different measured Vsini: $1.9,1.6,1.0$ and $8 \mathrm{Km} / \mathrm{sec}$ but same Li; also, the median Hyades $\mathrm{G}$ stars Vsini is $6 \mathrm{Km} / \mathrm{sec}$ and as shown in Figure 3, the difference in Li between these clusters is very small. I am not claiming that rotational velocity is not relevant in $\mathrm{Li}$ evolution, rather that its relevance is not yet well established and understood on observational grounds. 


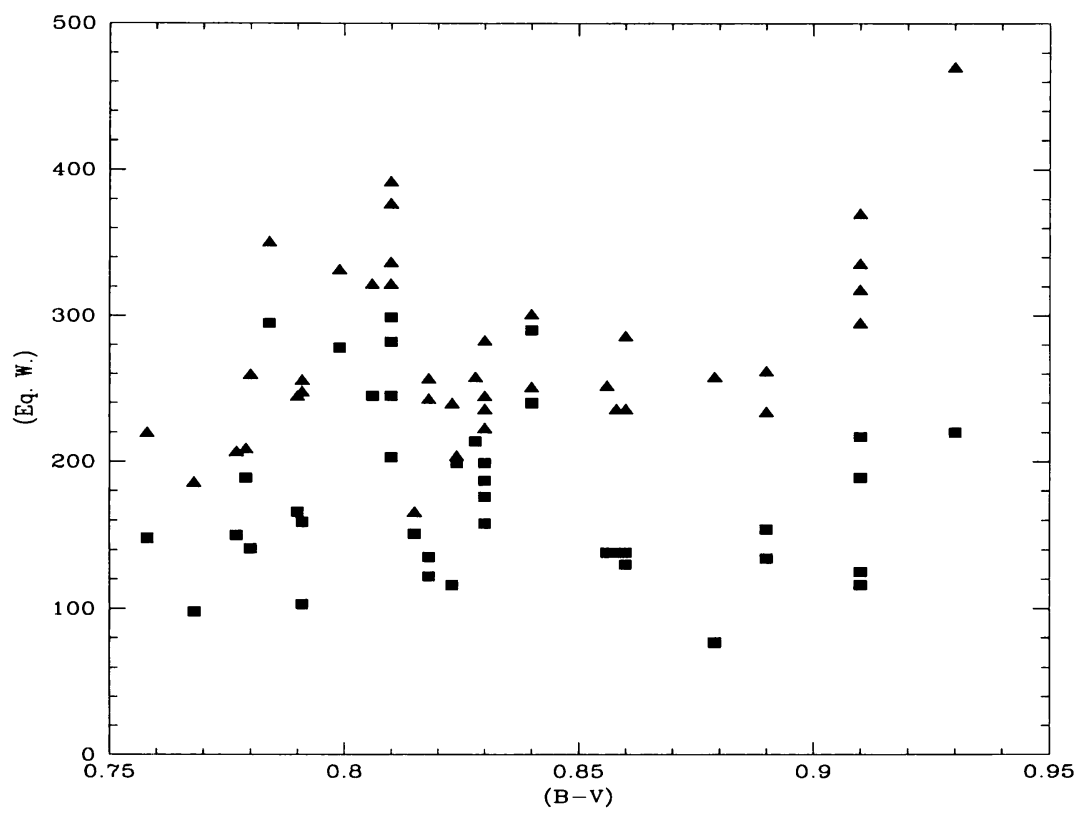

Figure 4. K equivalent widths vs. Li equivalent widths for Pleiades K stars, as measured by Jeffries 1999 and Soderblom et al. 1993

\section{Li Scatter?}

One advantage of studying stars in clusters is that we can assume a very simple population approximation: all the stars in a cluster share age, history and metallicity; they are perfect targets to investigate whether, at a given age and mass, all stars share the same $\mathrm{Li}$ abundance or not.

From Figures 1, 2 and 3 it is clear that almost no scatter is present (the definitive assessment on the presence of a small scatter would require a much better quality data sample) among $G$ stars belonging to one cluster for ages comprised between $30 \mathrm{Myr}$ and 1.8 Gyrs.

Yes, scatter is present among the G stars belonging to the 4 gyrs old M67. This fact is evident and it has been discussed by Pasquini et al. 1997. A couple of interesting points seem to emerge from the M67 data set: in $30-40 \%$ of the $\mathrm{G}$ stars a very strong depletion occurs, similar to that observed in the sun, and their $\mathrm{Li}$ content is at least 10 times lower than that of the other, otherwise similar objects. The second point is that almost no stars appear at intermediate Li values, suggesting that a bimodal distribution may be present and that the mechanism responsible for this extra depletion could be rather fast: an on-off type which occurs in some of the stars but not in the others. Note that this distribution is likely not peculiar to the M67 clusters, i.e. due to the cluster environment, because the same behavior is observed among G stars in the field, although these stars represent a much less homogeneous sample (Pasquini et al. 1994). 
As far as $\mathrm{K}$ stars are concerned, it is clear that a strong scatter is present in all clusters younger than the Hyades, therefore younger than $\sim 600 \mathrm{Myr}$. For older stars the Li abundance is so low that to obtain proper observations may become prohibitive.

\section{Pop I - Pop II Plateau Stars}

In the introduction I anticipated that the study of the Li evolution in Pop I stars has an impact on our understanding of the Li Plateau and the primordial nucleosynthesis. In this section I would like therefore to summarize how the above observations relate to this topic.

The fact that no PMS depletion is observed among metal rich stars is a strong support to the primordial interpretation of the Plateau, because PMS depletion could represent a strong Li sink, as well as very difficult to measure.

As far as MS depletion is concerned, the fact that $\sim 30 \%$ of the stars show extra depletion in M67, while a few 'outlayers' are found among the Pop II stars could also support the Plateau as signature of primordial Li. In fact this suggests that the depletion mechanism at its origin is strongly metallicity dependent, and therefore much less effective among the Plateau stars.

One result which I find rather annoying is that, once we have accepted that a number of 'outlayers' exists (even if their percentage is decreasing with metallicity), the upper envelope of the intermediate age and old clusters tends to flatten out for temperatures higher than $\sim 5800 \mathrm{~K}$, or, in other words, $\mathrm{Li}$ depletion tends to 'saturate' among old G stars. The early (and so far only!) observations of the very old NGC188 show stars with $\mathrm{Li}$ abundances comparable to the younger M67. I expect that a considerable fraction of very old, metal rich $\mathrm{G}$ stars still have still a $\mathrm{Li}$ abundance around $\mathrm{N}(\mathrm{Li})=2.1-2.2$.

The fact that 'standard' main sequence depletion is not strongly metallicity dependent, per se does not affect much the Pop II issue; however it shows clearly that current theories, which are the same predicting no Li depletion in Plateau stars, have some fundamental problem (see also Randich et al. 2000), and that the $\mathrm{Li}$ problem is not really understood. In order to advance, a more detailed work, similar to that performed for the Pop II stars, should be carried out, in order to define for the cluster stars accurate $\mathrm{T}_{e f f}$, and other elemental abundances like, e.g. $\mathrm{O}, \mathrm{Mg}$, Si. Very few data are available on old open clusters and on metal poor clusters! They are fundamental because they constitute the link between the Pop I and the Pop II stars. To understand if model atmospheres and stellar parameter determination are adequate is also fundamental, and, for instance Potassium spectra for $\mathrm{K}$ stars in young clusters could be essential. Finally, measurements of rotational velocities and magnetic fields proxies will help to shine some light on the role played by these parameters.

\section{Summary of Evidence}

Observations of $\mathrm{G}$ and $\mathrm{K}$ stars in Open Clusters start to provide basic constraints on Li evolution. While until a few years ago different sets of (mostly field ) data could provide different answers, now a quantity of well established 
observational evidence exists, and it should be considered when elaborating $\mathrm{Li}$ evolution theories.

- No strong PMS Depletion occurs among G stars; apparently with no strong dependence with metallicity.

- A Main Sequence 'normal depletion' exists: most of the action takes place between $\sim 100$ and $\sim 600 \mathrm{Myr}$, while it smooths considerably for older stars and may tend to saturation. No dependence of this depletion on metallicity is observed among $\mathrm{G}$ stars, while this may be present among the $\mathrm{K}$ stars.

- A Main Sequence 'extra depletion' is present among G stars: it affects up to $40 \%$ of the stars and takes place after $\sim 1.6$ Gyrs. It decreases the $\mathrm{Li}$ abundance of a factor $\sim 10$ or more. The distribution of $\mathrm{Li}$ abundance among old stars may be bimodal, indicating that this extra depletion occurs in a rather short period.

- For a given cluster, no large spread is detected for $\mathrm{G}$ stars younger than 1.6 Gyrs, but it is present at 4 Gyrs.

- Binarity (or something connected; magnetic activity?) may slow down considerably Li depletion.

Acknowledgments. I am indebted to V. Hill, R. Jeffries, R. Pallavicini and S. Randich for many discussions and for providing some of the data; to P. Bristow for a careful reading of the manuscript.

\section{References}

Balachandran, S., Lambert, D., Stauffer, J. 1996, ApJ 470, 1243 (B96)

Barrado Navasquez, D. et al. 1999, in "Cool stars stellar systems and the Sun XI ", Rebolo R. and Garcia-Lopez R. eds. in press. (BN99)

Boesgaard A.M., Tripicco, M.J. 1986, ApJ 303, 724

Boesgaard, A.M., 1987, ApJ 321, 967

Boesgaard, A.M. 1987, PASP 99, 1067 (B87)

Boesgaard, A.M. et al. 1988, ApJ 327, 389 (B88)

Boesgaard, A.M., Budge, K.G. 1988, ApJ 332, 410 (BB88)

Delyiannis, C. 2000, in "Stellar Clusters and Associations" R. Pallavicini et al. eds., ASP Conf. Ser. in press

Ford, A. et al. 1999, in "Cool Stars Stellar Systems and the Sun XI", Rebolo R. and Garcia-Lopez R. eds., in press. (F99)

Garcia Lopez, R., Rebolo, R, Martin, E. 1994, A\&A 282, 518 (GL94)

Hill, V., Pasquini, L. 1999, These proceedings (H99)

Hobbs, L.M.., Pilachowski, C. 1986, ApJ 309, L17 (HP86)

Hobbs, L.M. Pilachowski, C. 1986, ApJ 311, L37 (HP86a)

Hobbs, L.M., Pilachowski, C. 1988, PASP 100, 336 (HP88)

Hobbs, L.M. Pilachowski, C. 1988, ApJ , 334, 734 (HP88a) 
James, D.J., Jeffries, R.D. 1997, MNRAS 292, 252 (JJ97)

Jeffries, R.D. 1997, MNRAS 292, 177 (Je97)

Jeffries, R.D. 1999, in "Stellar Clusters and Associations" R. Pallavicini et al. eds., ASP Conf. Ser. in press

Jeffries, R.D., James, D.J. 1999, ApJ, 511, 218

Jeffries, R.D., James, D.J., Thurston, M.R. 1998, MNRAS 300, 550 (J98)

Jeffries, R.D. et al. 2000, in "Stellar Clusters and Associations" R. Pallavicini et al. eds., ASP Conf. Ser. in press (J99)

Jeffries, R.D. 1999, MNRAS 304, 821 (J99)

Jeffries, R.D. 1999, MNRAS 309, 189

Jones, et al. 1996, AJ 114, 352 (J96)

Jones B.F., et al. 1997, AJ 114, 352 (J97)

Jones, B.F, Fischer, D. Soderblom, D.R. 1999, AJ 117, 330 (J99b)

King, J.R. 1998, AJ 116, 254

King, J.R. Krishnamurthi, A., Pinsonneault, M.H. 2000, AJ 119, 859

Martin, E., Montes, L., 1997, A\&A 318, 805 (MM97)

Meola et al. 99, in "Stellar Clusters and Associations" R. Pallavicini et al. eds., ASP Conf. Ser. in press (M99)

Panagi, P.M., O’Dell, M.A. 1997, A\&AS 121, 213 (PO97)

Pasquini, L., Liu, Q., Pallavicini, R. 1994, A\&A 287, 191

Pasquini, L., Pallavicini, R., Randich, S. 1997, A\&A 325, 535 (P97)

Pasquini, L. 1998, in "Cool Stars Stellar Systems and the Sun X" A.K. Dupree and J. Bookbinder eds. CD-947 (P98)

Pasquini, L. 2000, in "VLT Opening Symposium" J. Bergeron ed., in press.

Pinsonneault, M.H. et al. 1989, ApJ 338, 424

Randich et al. 1997, A\&A 323, 86 (R97a)

Randich, S. et al. 1998, A\&A 333, 591 (R98)

Randich, S., Pallavicini, R., Mermillod, J.C. 1999 These proceedings (R99a)

Randich, S., Pasquini, L., Pallavicini, R. 2000, A\&A, in press (R00)

Russell, S.C. 1996, ApJ, 463, 593 (R96)

Soderblom, D.R. et al. 1990, AJ 99, 595 (S90)

Soderblom, D.R. et al. 1993, AJ 106, 1080 (S93)

Soderblom, D.R. et al. 1995, AJ 110, 729 (S95)

Soderblom, D.R. et al. 1999, AJ 118, 1301 (S99)

Spite, M., Spite F. et al. 1987, A\&A 171, L1 (S87)

Stauffer, J. et al. 1989, ApJ 342, 285 (S89)

Swenson, F.J. et al. 1994, ApJ 425, 286

Thorburn, J. et al. 1993, ApJ 415, 150 (T93) 